\title{
ADAM SMITH ET LA REPRÉSENTATION : DÉFENSE ET ILLUSTRATION DE LA MAIN INVISIBLE
}

Le propos de ce travail ${ }^{1}$ est limité à une question très précise : y a-t-il une théorie de la représentation chez Smith, et si oui quelle est-elle? On soutiendra que la façon dont Smith envisage la formation des connaissances est toujours semblable, quelle que soit leur nature ou la scientificité du propos. Et que l'unité de son œuvre tient dans cette similitude de traitement. Aborder l'œuvre de Smith de cette façon permet d'y découvrir une épistémologie subjective, où toute formation de savoir est aussi constitutive d'un sujet : théorie contradictoire avec cette neutralité objective qui dominera par la suite la philosophie des connaissances, et qui contribue encore à biaiser nombre de lectures sur Smith. On montrera d'abord comment une synthèse des réflexions menées par Smith sur les divers champs du savoir de son temps " révèle " une philosophie spéci-

1. Le matériel de travail utilisé pour cet article est « The Glasgow edition of the works and correspondance of Adam Smith. Commissioned by the University of Glasgow to celebrate the bicentenary of the Wealth of Nations " (1976). On en utilise surtout les trois moments principaux, avec leur abréviation consacrée : a) Essays on Philosophical Subjects (E.P.S.), issus des enseignements de Smith dans les années 1750, mais publiés à titre posthume par ses amis J. Black et J. Huthon, avec les commentaires de D. Steward (Londres, T. Cadell et W. Davies, 1795); b) the Theory of Moral Sentiments (T.M.S.), publiee d'abord en 1759 à Londres chez A. Millar, mais très largement révisée et augmentée au cours des deuxième et surtout sixième éditions, qui sert donc de réfërence (Londres, W. Strahan et T. Cadell, 1790); c) An Inquiry into the Nature and Causes of the Wealth of Nations (W.N.), publiée d'abord en 1776 à Londres chez W. Strahan et T. Cadell, et qui connut cinq éditions du vivant de Smith, peu modifies au-delà de la troisième, qui fait ici référence (Londres, les mèmes, 1784). Des références plus épisodiques seront faites aux trois autres volumes de l'édition de Glasgow, à savoir : Correspondance (vol. 6), Lectures on Jurisprudence (L.J., vol. 5) et surtout Lectures on Rhetoric and Belles-Lettres (L.R.B.L., vol. 4), qui comprend «A Dissertation upon the Origin of Languages » que Smith avait jointe à la troisième édition de T.M.S. Nous nous référons donc aux pages de l'édition de Glasgow, en les faisant précéder de deux numéros, qui indiquent le livre et le chapitre concemés pour W.N., ou la partie et sa première subdivision (section ou chapitre, selon les cas) pour T.M.S. ; pour E.P.S., la page est précédée d'un seul numéro, qui renvoie à chacun des différents essais, dans l'ordre où les présente l'édition de Glasgow. Seules les indications de pages sont portées pour les autres références.

Revue de synthèse: IV S. No 2, avril-juin 1989. 
fique de la connaissance qu'on peut qualifier après coup de représentative. On verra ensuite ce qu'apporte une application explicite de ce modèle formel aux deux domaines particuliers de la psychologie individuelle et du mécanisme social, dans lesquels Adam Smith s'est employé à produire lui-même des connaissances nouvelles. On conclura sur le nouveau statut ainsi conféré aux Essais philosophiques : non pas simple antécédent historique mais fondement logique duquel les autres moments de l'œuvre ne peuvent être séparés. Ce qui conduit à réinterpréter notamment le caractère autolimitatif généralement attribué à la " main invisible " dans la théorie du mécanisme social.

Après Smith, en effet, l'économie politique s'est développée en interprétant la main invisible comme une carence de l'analyse fondatrice, un vide qu'il convenait de combler par des analyses sur les conflits, le pouvoir et/ou les conventions, afin de mieux expliquer le passage entre le niveau individuel et le niveau social global. L'enjeu de ces «progrès analytiques " concerne le statut du discours par rapport aux phénomènes. Reste-t-il en extériorité, et conscient de l'être, en sorte que la constitution du sujet vivant (ses actions, ses jugements) demeure irréductible à tous les concepts imaginables de sujet théorique? Telle est, me semble-t-il, la démarche de Smith. Ou bien le discours sur le sujet s'objective-t-il au point de devenir lui-même un phénomène occultant toutes les autres composantes du sujet? C'est, à mes yeux, le parcours suivi après Smith par l'économie politique dont la fiction conceptuelle - de plus en plus cohérente et persuasive - tend à s'autoréaliser, à mesure où les sujets se transforment eux-mêmes en membres de la Société, agissant conformément au modèle de référence.

\section{1. - LA THÉORIE DES CONNAISSANCES COMME THÉORIE DU SUJET}

En prenant les Essais philosophiques pour base principale, on peut recenser méthodiquement l'ensemble des conditions qui paraissent nécessaires à Smith pour que se forment des connaissances, indépendamment du champ spécifique dans lequel elles s'expriment ${ }^{2}$. On consta-

2. Dans l'édition de Glasgow, l'agencement des thèmes des E.P.S. s'opère ainsi : 1, "The principles which lead and direct philosophical enquiries, illustrated by the history of astronomy "; 2 , "Illustrated by the history of the ancient physics "; 3, " The history of ancient logics and metaphysics "; 4, "Of the external senses "; 5, " Of the nature of that imitation which takes place in what are called the imitative arts "; 6, "On the affinity between music, dancing and poetry "; 7, "Of certain English and Italian verses ». La numérotation est de notre fait et vise à faciliter les références. 
tera que les traits essentiels de ce repérage renvoient à une véritable théorie de la constitution du sujet.

En se démarquant systématiquement d'une hypothétique vérité objective que posséderait la chose en soi, Smith pose que le sujet se construit d'abord une image des choses, en extériorité par rapport à lui, pour pouvoir ensuite penser les rapports qu'il entretient avec elles. C'est dans ce double mouvement de mise à distance, puis de mise en rapport que s'opère le processus d'identification à la fois du sujet et de l'objet : que vient à la conscience l'existence du réel objectivé. Il n'y a donc pas d'identité absolue des choses qui s'imposerait objectivement mais construction subjective, au contraire, de la nature des choses par un sujet qui se constitue lui-même à l'occasion de cette prise de conscience. L'identification n'est jamais donnée d'emblée, elle exige un travail; ce qui interdit de la ranger dans la catégorie des savoirs provenant de l'instinct comme «an impression immediately struck by the hand of nature, which does not wait for any recollection of past observation and experience" (E.P.S., 4-168).

\subsection{Les règles de construction de la représentation}

Tout acte de connaissance procédant par représentation se caractérise à la fois par la constitution d'une objectivité extérieure selon un processus interne au sujet, et par le repérage de la situation du sujet par rapport au temps et à l'espace de ces objets extériorisés. La première composante du savoir représentatif relève donc de la mise à distance de l'objet à connaître et que l'on fait exister à l'extérieur de soi. La seconde composante relève de la médiation qu'opère cette mise à distance, en ce qui concerne la constitution du sujet lui-même, et le retour sur soi des extériorités.

La représentation aura donc pour première règle de se construire à partir d'un processus unifié de distanciation-médiation; en sorte que toute connaissance de l'objet contient déjà un mode de reconnaissance du sujet. Mais le sujet ne peut précisément se reconnaître que si toutes les composantes de son être se trouvent impliquées dans ce processus. Et c'est la seconde règle assignée à la représentation, lorsque celle-ci concerne la connaissance du sujet.

Quels que soient les niveaux plus ou moins élaborés de cette connaissance du sujet par lui-même, elle requiert toujours l'unité de principe de ses composantes sensitives, émotives et rationnelles. Au niveau sensitif, le plus proche de l'animal, un savoir réglé par la représentation ne pourra donc exister sans un minimum de jugement, et aussi de raisonnement, ce 
qui le distingue du savoir instinctif de la pure intuition immédiate. Mais au niveau le plus spirituel, aucun savoir représentatif ne pourra non plus se former sans un minimum d'empirie, et aussi de jugement, ce qui le distinguera du pur savoir conceptuel qui exclut a priori toute composante irrationnelle. Il suit notamment que la connaissance représentative ignore par construction cette opposition du normatif et du positif qui est au fondement du savoir conceptuel. Car ce mode de connaissance représentative possède en soi une vertu dynamisante pour le sujet qui s'y implique toujours entièrement.

\subsubsection{La règle de distanciation}

Le travail de formation des connaissances peut s'effectuer d'après Smith selon trois procédures : par observation, par jugement et par abstraction. La première de ces procédures s'initie dans les sens externes et la deuxième dans une sensibilité interne, qui provoque un sentiment de plaisir ou de dégoût, qu'on repère particulièrement bien dans l'émotion artistique; la troisième s'initie dans le raisonnement.

Au niveau d'un savoir provenant des sens externes, c'est le contact direct qui révèle à la conscience du sujet cette « substance tangible " des choses (E.P.S., 4-159) qui lui sont indépendantes. La résistance physique au toucher est ainsi primordiale pour établir la consistance des choses et leur extériorité. Pour les autres sens, le travail de distanciation est déjà plus élaboré. Si le goût est une combinaison de toucher et d'effet spécifique, le son, la couleur, l'odeur n'ont aucun lien avec le toucher, ni aucune ressemblance avec la consistance de la matière. Des vibrations, des images... "représentent" (E.P.S., 4-151) ici les objets tangibles à partir des impressions laissées sur la rétine, le tympan. On prend l'habitude d'extérioriser la cause et de ne pas l'imputer à la transformation de l'organe lui-même. Pour ces autres sens, c'est donc la distance spatiale qui est significative de l'extériorité du sujet à l'objet.

La sensibilité interne fait partie du processus de formation des connaissances, puisqu'elle permet d'accéder à la beauté et à la vérité des choses, qui sont des qualités intrinsèques que leur confère la nature. Or, le plaisir imprévu ("surprise ») provoqué par l'art de la peinture, de la sculpture, de la danse ou de la poésie provient de cet effet de distance que sait laisser l'artiste entre le modèle et sa représentation (E.P.S., 5-183). L'œuvre ne prend son véritable sens que dans cette "étrangeté " par rapport à la nature qu'elle évoque. En tant qu'objet lui-même, le tableau, la statue, etc., ne se confondent pas avec l'objet qu'ils représentent et n'en sont pas de simples copies. L'émotion produite par la reconnaissance de la beauté dépend de cet intervalle par rapport à l'objet représenté. La musique est ainsi la limite des arts imitatifs, ne reflétant 
pratiquement aucun objet déjà donné dans la nature, mais produisant directement son effet originel (E.P.S., 5-206).

Quant à la formation de connaissances à partir du raisonnement, une incursion dans la théorie du langage fera bien apparaître cette extériorisation des choses par rapport au sujet qui les pense. Car le langage ne se trouve pas déjà donné dans la nature des choses; celles-ci ne parlent pas d'elles-mêmes. "If custom had so ordered it, [the words] might with equal property have been made use of to express any other meanings of ideas " (E.P.S., 4-157). Les objets sont exprimés par des sons qui en constituent des représentations arbitraires, que le sauvage se forme instinctivement dès l'origine pour communiquer socialement son rapport avec les objets qui l'entourent et rendre intelligibles ses besoins (L.R.B.L., 203). Si les premiers sons ont pu participer du langage de la nature (E.P.S., 4-161), ils s'en sont rapidement autonomisés pour ne plus s'articuler que selon des règles d'analogie sonore. Le fondement grammatical de toutes les langues tient ainsi dans le plaisir mélodique de l'oreille (L.R.B.L., 211) et relève en somme de l'art musical dont on vient de dire qu'il est l'un des moins imitatifs.

Au départ, chaque événement particulier est dénoté par un mot particulier qui le désigne totalement; mais l'infinité des cas surcharge la mémoire et pousse à la décomposition de l'événement en ses divers éléments (ses "composantes métaphysiques", L.R.B.L., 217). Un processus d'abstraction progressive est ainsi généré, qui part des observations concrètes et met de plus en plus de distance entre la situation observée et sa représentation. Les diverses langues sont alors devenues des "machines" de plus en plus complexes, composées d'éléments arbitraires de plus en plus simples (L.R.B.L., 224).

\subsubsection{La règle de médiation}

La mise à distance des objets de la connaissance ne fait que préparer le moment crucial de leur réappropriation par le sujet, qui va maintenant se situer lui-même dans ses rapports aux objets. Car l'extériorisation première n'a de sens que si elle fonctionne tout à la fois comme prise de conscience du sujet par lui-même. Dans cette médiation, on peut lire chez Smith une véritable théorie de la constitution du sujet, qui s'élabore dans les rapports que le sujet entretient avec le temps et avec l'espace de sa représentation des objets.

La constitution du sujet dans sa dimension temporelle provient d'abord de l'observation et de la connaissance sensible des objets qui l'entourent. Elle résulte d'un effet de mémoire qui met en rapport le cas présent avec un cas antérieur dont le sujet a eu l'expérience. Seule la répétition dans le temps des cas particuliers permet d'établir les pre- 
mières règles générales de la perception (E.P.S., 4-139). Et la connaissance des objets apparait toujours comme une reconnaissance de leur identité à travers les formes pourtant variables de leur apparition concrète (E.P.S., 4-155). S'il est possible de définir le mouvement comme une caractéristique tangible des choses, c'est parce que la mémoire nous a donné l'expérience d'un rapport entre le temps substantiel des objets et le temps propre du sujet.

Mais la connaissance du temps vient aussi à la conscience du sujet au niveau de sa sensibilité interne, par l'appréciation qu'il porte sur les œuvres d'art. Non pas le temps répétitif du redoublement des copies, mais le temps propre de l'œuvre qui lutte contre le temps. Adam Smith évoque ici la différence entre le tableau et un miroir qui ne fait que représenter l'objet présent, incapable par le fait de créer l'extériorité nécessaire du reflet vis-à-vis de son modèle (E.P.S., 5-185). Il accorde d'ailleurs une qualité émotionnelle toute particulière à l'art musical, capable de construire son temps propre. Dans chaque cas, c'est donc la prise de conscience de l'existence d'un artiste dans l'œuvre qui renvoie au temps du sujet.

Au troisième niveau de la formation des connaissances, la conscience du temps se loge aussi dans les constructions abstraites qu'élabore le sujet à propos des phénomènes. La "Dissertation sur l'origine des langues " et l' " Histoire de l'astronomie " montrent bien comment les formes abstraites se sont élaborées dans l'imagination, à partir de l'abandon progressif des références aux observations sensorielles.

« As neither quality nor relation can exist in abstract, it is natural to suppose that the words which denote them considered in concrete, the way in which we always see them subsist, would be of much earlier invention than those which express them considered in abstract, the way in which we never see them subsist " (L.R.B.L., 206).

Il en est de même dans la généalogie des corps célestes, qui aboutit à la gravitation universelle au terme d'un long cheminement commencé avec les sphères solides concentriques (E.P.S., 1-49).

Mais par-delà cette relativité historique des savoirs théoriques, qui dépendent des circonstances de leur élaboration, c'est la question des rapports du sujet avec le temps qui est ici posée. Car le temps en vient lui-même dans l'histoire à faire l'objet de représentation théorique. Il devient conscience du temps, sous la forme d'une suite d'événements se succédant linéairement de manière plus ou moins prévisible. Avec les progrès de l'abstraction raisonnante, le temps se transforme en construction de l'esprit qui permet aux événements d'être déjà dans l'imagination, avant de survenir effectivement. 
" As the ideas which represented such a train of things would seem all mutually to introduce each other [...] so, when the objects themselves occur, every last event seems in the same manner to be introduced by the foregoing, and introduce the succeeding " (E.P.S., 1-41).

Et toute interruption de la chaîne par un événement fortuit requiert un nouvel effort d'abstraction permettant de refaire le lien temporel. Au final, l'historicité du savoir serait en quelque sorte produite par le mouvement même de la conceptualisation du temps.

Pour en venir à la prise de conscience de la situation du sujet dans l'espace, elle résulte de cette même médiation par les objets de la connaissance, et opère aussi à chacun des trois niveaux physique, psychique et intellectuel qui constituent le sujet.

Comme dans la formation des connaissances à propos du mouvement, ce sont d'abord les expériences du toucher qui font découvrir les caractéristiques spatiales de la matière : son étendue, sa forme et sa divisibilité (E.P.S., 4-137). Et il faut au sujet une bonne dose d'apprentissage et de mémoire pour que les sons, les odeurs ou les images soient imputés à ces qualités tangibles de l'espace matériel, plutôt qu'à une modification des organes sensoriels eux-mêmes. Un an sera nécessaire à l'opéré de la cataracte pour qu'il reconstitue son sens de l'espace; au début, il a l'impression que les objets touchent ses yeux (E.P.S., 4-159). L'intensité du plaisir que l'on peut prendre à l'admiration des œuvres d'art n'est pas elle-même indépendante des rapports des objets dans l'espace; Smith souligne ainsi qu'une statue plaira davantage qu'un tableau par la possibilité d'une pluralité des points de vue (E.P.S., 5-186).

Mais c'est encore avec l' "Histoire de l'astronomie " que l'on saisit le mieux comment l'espace (planétaire, en l'occurrence) n'est pas qu'une caractéristique inhérente aux objets dont on fait progressivement l'expérience; qu'il est aussi une notion permettant au sujet de se situer abstraitement dans un système de places assignées plus ou moins harmonieusement aux choses. Et la philosophie y est explicitement définie comme système de relations entre les choses de la nature, destiné à créer de l'ordre dans le chaos des apparences (E.P.S., 1-45). Idée qui se prolonge dans l' " Histoire de la physique ancienne " : là où l'ignorance et la superstition engendraient l'espace morcelé du polythéisme, sans principe directeur, la science produit la représentation d'un espace cohérent, gouverné par des lois générales et reproductibles dans le temps.

" In the first ages of the world [...], the idea of a universal mind of a God of all who originally formes the whole and who governs the whole by general laws, directed to the conservation and prosperity of the whole, without regard to that of any private individual, was a notion to which they were utterly strangers " (E.P.S., 2-112, 113). 
L'esprit universel du monothéisme, c'est pour ainsi dire l'espace en tant qu'abstraction.

\subsection{La corrélation des procédures de connaissance}

C'est bien parce qu'elle est essentiellement rapportée à une théorie du sujet que la formation des connaissances s'analyse toujours chez Smith dans son aspect tripolaire : aucun rapport physique aux objets qui ne soit connecté aux émotions et aux concepts, et réciproquement. Car ces trois moments sont toujours nécessaires à la constitution d'un sujet qui se pense comme tel : esprit, âme et corps à la fois. Et l'erreur pourrait se caractériser chez Smith comme un effet de connaissance partielle ou fallacieuse, produit par l'inachèvement du processus de constitution du sujet lui-même. Quel que soit le niveau du savoir concerné, l'erreur signale un manque dans ses procédures de formation subjective; elle signifie que l'une au moins de ses composantes, sensorielles, émotionnelles ou rationnelles, fait défaut. On citera par exemple, le défaut de raisonnement dans l'impression ressentie par l'opéré de la cataracte précédemment évoqué, un manque de jugement dans l'appréciation des cuvres d'art en fonction du marché (E.P.S., 5-183) et un manque de concret dans la métaphysique idéaliste qui « embrouille tout sans rien expliquer " (E.P.S., 4-140) avec ses illusions d'essence des choses (cf. les idéaux platoniciens) :

"As the objects of sense were apprehended to have an external existence, independant of the act of sensation, so these objects of the understanding [the specific essences of things. L. G.] were much more supposed to have an external existence independent of the act of understanding " (E.P.S., 3-121).

Cet acte de compréhension ne se limite, chez Smith, ni à l'enregistrement de signaux sensibles, ni à la production de formes intelligibles car elle est à jamais inséparable d'une charge de plaisir. Ce plaisir correspond à un instinct vital de connaitre qui, d'une part, dynamise la formation des connaissances dans une relation effectivement vécue par le sujet et qui consacre, d'autre part, ce sujet dans son statut d'objet de la nature, réalisant malgré lui une volonté sacrée qui le transcende.

Au premier niveau de l'observation des phénomènes de la nature, on est d'emblée "étonné " par la non-coïncidence des sensations issues d'un même objet; les sons, les goûts, les odeurs ou les images n'ont rien à voir avec les qualités tangibles que révèle le toucher; et ils n'ont rien à voir entre eux non plus. Mais on est porté d'instinct à organiser rationnellement ces multiples sensations (E.P.S., 4-153), et à rechercher expérimentalement un principe de connexion qui unifie leur sens, au-delà 
d'une simple rémanence d'impressions sensibles. C'est de la réflexion sur les diverses apparences concrètes que proviennent donc les connaissances sur la substance des choses; ce qu'indique, par exemple, l'apprentissage de la perspective.

On notera qu'une réflexion qui se limiterait aux seuls mots ne peut produire cet effet en raison de la médiation artificielle qu'ils introduisent par rapport à la nature : un raisonnement sur les mots ne peut que tourner en rond (E.P.S., 3-125). Par contre, les différentes formes d'expression du langage de la nature ne peuvent pas ne pas posséder certaines « analogies " entre elles. Ainsi, " the visible character which represents to our eyes the tangible globe could not so well represent the tangible cube " (E.P.S., 4-158). Or, les sens vivent directement le langage de la nature, et c'est de cette communion que peut naitre une réflexion véritable sur les choses.

Le principe de plaisir qui se mêle toujours à l'apprentissage du raisonnement est ici particulièrement lisible, car ce niveau primaire des sensations est le lieu privilégié des désirs et de leurs satisfactions ou de leurs frustrations. C'est dans ce contexte que l'instinct reçoit d'ailleurs sa définition explicite (E.P.S., 4-168, supra), comme impression d'origine naturelle et non expérimentale, avec cette précision :

" all the appetites which take their origin from a certain state of the body seem to suggest the means of their own gratification; and even long before experience some anticipation or preconception of the pleasure which attends that gratification " (E.P.S., 4-165).

Comme le montrent les comportements buccaux et sexuels du nourrisson, c'est l'instinct vital qui organise les premières perceptions autour d'un principe unifiant, qui nous sécurise et nous fait plaisir. Les sens externes semblent ainsi nous avoir été donnés :

« in order to inform us concerning that situation [...] of those external bodies which [...] may sooner or later affect our situation and eventually either benefit or hurt us" (E.P.S., 4-168).

Au niveau maintenant des effets émotifs produits par la contemplation des situations extérieures (spectacles, tableaux...), Smith évoque «that fellow-feeling which Nature has, for the wisest purposes, implanted in man " (E.P.S., 5-199). Cette notion, qualifiée aussi de "sympathie " visà-vis des objets représentés, renvoie au mécanisme central de la Théorie des sentiments moraux, qui fait de l'émotion le fondement essentiel du sens moral. Avant d'approfondir cette question plus loin, indiquons brièvement qu'il s'agit d'une caractéristique de sensibilités propres à la nature humaine et qui porterait chacun de nous spontanément à se 
positionner du point de vue de l'autre, et à juger les propriétés morales de nos propres actions à partir de ce positionnement. C'est dans une projection de ce type que s'élabore le goût artistique, selon ce qu'on imagine du modèle représenté, et selon le degré d'approbation que sa représentation provoque. A l'inverse, il suffit que l'instinct de sympathie n'accompagne pas le jugement esthétique pour que le goût se dénature, jusqu'au point où la mode ou l'orgueil fasse apprécier les cuvres (antithèses, rappelons-le, des copies reproductibles) sur la base de critères marchands (E.P.S., 5-183).

Mais la composante sensitive n'épuise pas cependant le mécanisme de connaissances qui s'initie dans la contemplation des œuvres d'art. La dénomination "d'arts imitatifs" indique d'abord assez bien que la connaissance qui se forme ici est une reconnaissance de situations ou d'objets observés initialement dans la nature. La sensation émotive renvoie donc toujours à quelque impression sensorielle préalable. Mais il y a plus, car elle ne peut survenir qu'en raison d'une prise de conscience du pourquoi de son existence. Nous sommes véritablement " heureux » de comprendre comment l'effet de surprise résulte de la distance entre le modèle et sa représentation et des rapports que l'artiste introduit dans l'intervalle qui sépare ces deux genres totalement différents que sont le tableau comme objet et l'objet du tableau (E.P.S., 5-185). A l'inverse, on comprend aussi pourquoi dans le « trompe-l'œil ", ou dans la copie qui peut se prendre pour l'objet qu'elle représente, l'effet d'illusion, ou l'effet répétitif (le «miroir ») ainsi créés ne procurent aucune émotion vraie. A la limite, l'imitation servile - du type projection de neige en papier à l'opéra - devient tout à fait ridicule (E.P.S., 5-201).

$\mathrm{Au}$ niveau le plus abstrait de la formation du savoir, les systèmes d'explication philosophique des phénomènes naturels n'ont pour Smith qu'une vérité relative, qui dépend de leurs conditions d'existence et particulièrement de la place du sujet dans la formation des connaissances, tandis que l'histoire des savoirs n'est qu'une longue suite de transgressions de ces représentations. Il n'y a donc pas de principes innés de la connaissance qui préexisteraient dans l'entendement humain, puisque les catégories de la raison se constituent, au contraire, à l'occasion des expériences vitales du sujet. L'observation et l'émotion, qui situent le sujet dans la nature, conditionnent ainsi la formation de son savoir théorique.

Les conditions du savoir qui relèvent de l'observation concernent tout à la fois le réalisme des hypothèses et la vraisemblance des résultats. Ainsi, à propos des conséquences logiques que pourrait avoir un manque hypothétique de sens du toucher (E.P.S., 4-150), « it is unnecessary to throw away any reasoning or to hazard any conjectures about what might 
be the effect of what I look upon as altogether an impossible supposition ". Quant à la vraisemblance des résultats, c'est une nécessité de la connaissance théorique si elle veut s'imposer. Pour que chacun puisse se persuader que la représentation proposée correspond bien à la vérité de la nature, et donc à la réalité, il lui faut la percevoir comme système d'analogies avec les expériences de la vie courante (E.P.S., 1-105).

Mais l'originalité principale de l'épistémologie smithienne vient de la considération des critères de sensibilité pour estimer les systèmes d'abstraction, envisagés du point de vue de l'œuvre, et donc explicitement comme systèmes de représentation. "The first perceptions, as well all other experiments upon which any general rules are founded cannot be the object of reason but of immediate sense of feeling " (T.M.S., VII 3319). Et le jugement qu'on porte sur nos raisonnements est toujours finalement lié aux émotions qu'ils suscitent (E.P.S., 1-48); la vérité des choses ne se résume pas à leur concept, car le sujet vivant reste l'ultime réfërent de son propre savoir.

Deux composantes de cette charge émotionnelle sont repérables dans la formation des systèmes philosophiques que Smith étudie à propos de l'astronomie : une composante rassurante et une composante stimulante, qui sont chacune au fondement de l'action des sujets. La raison philosophique en se proposant de " recoller, dans l'imagination, les morceaux " d'une nature apparemment menaçante ne fait qu'exercer une fonction précédemment remplie par la superstition; et la différence n'est nullement une question d'adéquation aux faits. Car une histoire des systèmes philosophiques peut s'écrire en fonction de leur faculté plus ou moins grande à exciter l'imagination et à susciter l'admiration. La formation des connaissances résulte d'un ensemble d'artifices, mis en œuvre comme au spectacle :

" without regarding their absurdity or probability, their agreement or inconsistency with truth and reality, let us [...] content ourselves with inquiring how far each [of the different systems of nature. L. G.] are fitted to soothe the imagination, and to render the theatre of nature a more coherent and therefore a more magnificent spectacle than otherwise it would have appeared to be $"(E . P . S ., 1-46)$.

Mais cette imagination créatrice, qui résulte de nos peurs instinctives, tend naturellement à se justifier et à s'expérimenter concrètement, à prendre autrement dit, un contenu pratique : " mankind have had at all times a strong propensity to realise their own abstractions " (E.P.S., 3125). De sorte que la vérité des systèmes philosophiques se juge aussi à leur capacité de transformation des substances spéculatives en substances vivantes : à la qualité stimulante de leurs catégories par rapport aux actions des sujets. 
$\mathrm{Au}$ terme de ces premiers développements, il nous a donc paru possible de dégager, dans le texte philosophique de Smith, les principes d'une théorie de la formation des connaissances qui fonctionnerait comme représentation, à mi-chemin en quelque sorte de la révélation et de la conceptualisation. Représentation qui se caractériserait alors par un système imaginaire que se construit le sujet pour prendre conscience des choses, et aussi conscience de cette conscience pour intervenir sur ces choses. Il s'agit maintenant de s'interroger sur la théorie du sujet, qui est pris comme objet de connaissances dans les écrits ultérieurs de Smith et de vérifier que c'est bien une théorie implicite de la représentation qui est à l'œuvre dans l'élaboration de ces textes positifs.

On le vérifiera en répondant à ces deux questions : a) comment le sujet peut-il théoriquement se dédoubler dans un processus complet de distanciation médiatrice, pour se constituer à la fois comme objet et sujet, et par rapport à quelles normes d'espace et de temps; b) comment une telle connaissance théorique du sujet (à un niveau le plus élaboré des savoirs donc) peut-elle incorporer tout de même les dimensions phénoménales de la sensation et du jugement qui sont propres à chaque individu particulier?

En tant qu'objet de savoirs théoriques, le sujet apparaît essentiellement, chez Smith, sous une double forme. La première est théorisée dans la Théorie des sentiments moraux (T.M.S.), où « l'instinct de sympathie " nous paraît exactement jouer le rôle d'objet de la représentation, par rapport auquel s'effectue le travail d'extériorisation-médiation. Mais le sujet est théorisé sous une seconde forme dans la Richesse des nations (W.N.), où c'est "l'instinct d'échange » qui nous paraît jouer ce rôle d'objet de représentation. D'emblée, il faut donc remarquer que ces deux instincts sont de nature spécifique et qu'ils déroulent leurs effets dans des champs ayant leur logique propre. L'ordre de leur présentation est circonstanciel, et sans implication théorique.

\section{2. - LA REPRESENTATION THÉORIQUE DU SUJET SOCIAL}

Les premiers développements de la Richesse des nations sont consacrés aux données expérimentales du social. Ce qu'on ressent, ce qu'on observe à son propos pourrait se résumer par ce constat : un instinct d'échange fonde la division du travail qui est source de la richesse.

On observe d'abord une grande différence dans la quantité de choses que chaque homme peut consommer d'une nation à l'autre; on observe 
aussi de fortes disparités dans l'usage du travail national, qui peut être divisé en un nombre plus ou moins grand d'activités interdépendantes. Là où cette division est bien établie, chacun travaille essentiellement pour les autres, et devient une sorte de marchand : la société est à proprement parler devenue une société commerciale (W.N., I 4-37). Chacune des choses qui font l'objet du commerce possède à chaque instant un prix qui lui est propre, qui s'impose à tous par l'intermédiaire du marché, et qui s'exprime sous forme de quantité de monnaie nécessaire pour l'acheter.

Dans le même temps, chaque homme ressent instinctivement en lui une disposition naturelle à échanger des choses avec ses semblables. Cette propension lui procure un plaisir spécifique, étranger au monde animal, et indépendant de la satisfaction matérielle du besoin lui-même (W.N., I 2-29). " This division of labour, from which so many advantages are derived, is not originally the effect of any human wisdom, which foresees and intends that general opulence to which it gives occasion" (W.N., I 2-25). Pourtant, chacun ressent bien que cet instinct d'échange est finalisé par la recherche de son propre intérêt - sans considération sincère pour le désir de l'autre - et qu'il opère selon des conventions où n'entre aucun lien affectif (T.M.S., II 2-86). Comment expliquer alors cette étrangeté d'une société qui ne se fonde pas sur la bienveillance altruiste et qui ne recourt pas cependant au vol ou à la violence pour subsister? Telle est la question constitutive d'une théorie du sujet social. Théorie dont il faut se demander si elle s'élabore bien chez Smith selon les principes de la représentation, avec ses deux règles constitutives (distanciation/médiation), et sa règle de fonctionnement, en tant que support d'action (par la corrélation qu'elle établit entre les diverses formes de connaissance).

\subsection{De l'instinct d'échange à la justice dans l'échange}

L'essence caractéristique du sujet social qu'il s'agit maintenant de représenter est donc son instinct à l'échange. Or, l'instinct suppose par définition une préconception informelle du plaisir qui précède toute expérience de sa réalisation (E.P.S., 4-165). Par nature, il s'oppose à toute représentation directe, en sorte qu'un déplacement de l'objet d'analyse est ici nécessaire pour prendre connaissance du sujet social. Dans la Dissertation sur l'origine des langues, on a déjà vu comment les mots socialisent les besoins; ils ne suffisent pas pour autant à constituer le sujet social. Car cette représentation des besoins opère selon une règle d'harmonie des sonorités qui ne saurait contribuer à la satisfaction des besoins eux-mêmes. Il en va de même pour le prix des choses qui règle 
nominalement la pratique des échanges quotidiens; car ces prix nominaux expriment les phénomènes courants dans un langage commode, sans révéler d'aucune façon la logique de l'échange elle-même.

\subsubsection{Le moment de la distanciation}

La nouveauté imaginée par Smith consiste à centrer toute l'analyse sur la notion de justice dans l'échange, qui devient du coup le véritable objet théorique de la représentation du sujet social. Cette justice s'apprécie par l'égalité des valeurs échangées entre marchands et cette égalité opère par suite d'un accord mutuel sur les valeurs échangées. Avec cette définition préalable de la valeur comme pouvoir conféré par un bien que l'on possède dans l'acquisition d'un bien que l'on ne possède pas; le prix étant la mesure de cette valeur relative entre les biens (W.N., I 5-47).

On a d'abord l'impression que c'est en soi que repose naturellement la justice des échanges, qui aurait le statut de connaissance instinctive, sans aucun travail de reconnaissance à effectuer. Mais ceci est une illusion, comme l'indique bien l'ensemble des prix qu'on observe communément sur le marché des choses qui s'échangent. Un tel prix mesure le pouvoir d'acquérir de la monnaie par la vente d'un bien que l'on possède; il fait l'objet de marchandages continuels et s'établit en fonction des seuls intérêts personnels. Une adhésion commune à ces prix requiert donc une " convention sur les valeurs " qui, seule, peut garantir que la société marchande se reproduira à l'abri des agressions mutuelles contre la personne et les biens de chacun : c'est-à-dire selon un principe de justice (T.M.S., II 2-86). Or, la logique de cette convention n'est pas directement lisible dans les apparences du marché, qui fonctionne comme un vaste jeu de miroirs déformants, où ne s'affichent pas directement les désirs de chacun, mais les prétentions que chacun a de satisfaire les désirs qu'il suppose à l'autre. Cette logique particulière du désir n'est pas arbitraire, puisqu'elle réalise les finalités suprêmes que la nature a mises en l'homme, en lui faisant désirer sa conservation et son identification propre. Du moins cette logique ne relève-t-elle pas de l'entendement puisqu'elle s'opère d'instinct, spontanément, et comme à notre insu. Pour comprendre la logique de la convention sociale un déplacement de champ est donc nécessaire ${ }^{3}$; pour élaborer une théorie de la socialisation, il faut d'abord construire une image des choses qui s'échangent, en extériorité par rapport au sujet marchand. Et c'est ce que propose la nouvelle représentation économique qui commence par extérioriser la

3. Notons donc la spécificité du savoir représentatif, par opposition à l'intuition " réaliste ", qui chercherait ici à déchiffrer la signature du grand mécanicien, sous l'hypothèse que toutes les parties de la machine portent en elles leur signification ultime, accessible par l'entendement. 
justice en la faisant fonctionner d'elle-même dans l'échange, comme un mécanisme objectif, extérieur aux subjectivités individuelles donc, et sans que personne n'y mette du sien.

Il s'agit donc de prendre d'abord du recul par rapport aux impressions de nos sens, qui appréhendent spontanément les échanges marchands à travers un ensemble de prix nominaux. Ce moment de la distanciation s'exprime chez Smith par l'image du prix réel, défini par le pouvoir que confère un bien dans l'acquisition du travail d'autrui ; ou réciproquement, par la quantité de travail cédé pour acquérir ce bien (W.N., I 5-47).

Pourquoi le travail? Parce que c'est une image transhistorique qui garde le souvenir de ce temps où l'activité humaine a commencé de se diviser entre ce que l'on fait pour soi et ce que l'on fait pour autrui. Le travail cédé indirectement dans l'échange fonde la valeur de la chose acquise, comme si l'on avait dû se la produire à soi-même. En ce sens, le travail est le signe de l'échangeabilité des choses. Et de même que dans l'analyse morale, la vertu d'une action s'apprécie à la conformité de sa nature par rapport aux motifs qui l'ont provoquée (T.M.S., I 0-26), de même pourrait-on dire que la valeur sociale de toute chose découle de sa propriété de convenance à l'échange : sa capacité, autrement dit, de renvoyer à une implication personnelle de chacun dans son rapport à l'autre. Car l'échange de quelque chose est toujours cession d'un travail qu'on n'a pas mis en œuvre pour soi. L'apparence des intérêts personnels pour les choses qui s'échangent cache donc un échange de travaux particuliers entre les sujets vivants, qui sont au fondement des connexions sociales. Comme si l'instinct de sympathie que le sujet vit concrètement au plan moral servait de référent imaginaire pour procéder aux abstractions qui vont rendre compte du sujet social. En quoi on peut considérer que se trouve satisfaite la règle de réalisme dans les hypothèses.

Mais le travail n'a pas pour seule caractéristique de conserver la trace indélébile des origines du social, il est aussi le seul moyen de mesurer l'accroissement des richesses depuis que le travail a commencé de n'être plus mis en œuvre seulement par soi-même, mais par l'intermédiaire de propriétaires fonciers et mobiliers, à qui revient naturellement une part des richesses produites de cette façon. La question de la mesure adéquate des valeurs est d'ailleurs déterminante dans le choix du signe représentatif des richesses qui s'échangent. Et les variations dans les conditions de production du métal précieux (W.N., I 5-49), autant que les manipulations officielles des princes (W.N., I 4-43) empêchent qu'on puisse faire notamment de la monnaie une mesure correcte de la valeur des choses, ou a fortiori le pivot représentatif d'une socialisation équitable (L.J., 101).

Avec l'unité de travail, au contraire, il est possible de poser abstraitement et sans trop d'arbitraire par rapport au sens commun que la variété 
empirique des travaux concrets peut s'exprimer dans une même unité comptable qui les résume tous (W.N., I 5-48), et qui reste, pour chaque travailleur, identique à elle-même à travers les variations de son activité laborieuse (W.N., I 5-50) : «Equal quantity of labour at all times and places may be said to be of equal value to the labourer. "

Ce principe d'identité préliminaire ( $\mathrm{A}$ est $\mathrm{A}$ ) indique bien le statut du prix réel dans la représentation des échanges. Il s'agit d'une construction imaginaire permettant de dépouiller les phénomènes observables de leurs particularités circonstancielles. Dans le processus de connaissance représentative du sujet social, le prix réel joue donc la fonction du concept par rapport à la notion immédiate du prix nominal. Il est l'abstraction nécessaire (W.N., I 5-49) à partir de quoi les phénomènes et les comportements de l'échange peuvent prendre la signification d'un processus de socialisation.

A défaut de concevoir l'existence du prix réel, les sujets peuvent sans doute être le jouet d'un mécanisme social, du moins ne peuvent-ils pas en avoir une juste conscience, ni donc agir sur lui efficacement. Ils restent prisonniers d'une préconception de relations duelles ou institutionnelles, vécues de façon circonstancielle et plus ou moins arbitraires. Car la justice des échanges ne parle pas naturellement d'elle-même dans les prix nominaux, quand bien même seraient-ils contractuels. Ceux-ci ne donnent que l'intuition de la valeur des choses et pour qu'un savoir se constitue à son propos, encore faut-il que se forme dans la conscience une image susceptible de représenter cette valeur : telle est la fonction assignée par Smith au prix réel.

\subsection{Le moment de la médiation}

Pour que la théorie qui est en train de s'élaborer à propos du sujet social opère bien sur le mode de la représentation, il est nécessaire que cette équité objective des échanges marchands qu'on vient d'extérioriser (sous la forme d'échange de travaux) fasse maintenant retour sur le sujet et le constitue comme tel. Et c'est ce qui, dans les savoirs, distingue le mode représentatif du mode conceptuel : le mécanisme socialisant ne peut jamais s'y objectiver en s'autonomisant par rapport aux sujets qui le mettent en œuvre. Un moment de conscience explicite est ici requis, qui interdit au sujet social d'être un objet malgré lui (ce qui fonde d'ailleurs la critique smithienne de Mandeville, T.M.S., VII 2-308).

Ce moment de la médiation va prendre chez Smith la forme du rapport entre le prix réel de chaque marchandise et son prix naturel, défini comme la récompense normale de ceux qui ont permis sa mise en vente. 
Après avoir mis à distance l'expérience sensible des désignations nominales, il s'agit maintenant de reconnaître, sous l'apparence des prix bilatéraux, la juste rémunération qui est due à chacun. En somme, là où l'image du prix réel était une condition suffisante à la connaissance des échanges matériels, sa médiation par les prix naturels figure la condition nécessaire pour connaître les sujets de l'échange eux-mêmes, en tant qu'objet du processus social.

La procédure complète de représentation ne peut alors faire retour sur le concret des prix de marché que s'ils sont constamment référés à leur norme naturelle. Et c'est la fonction attribuée au marché concurrentiel : fonctionnement social qualifié de normal, dans la mesure où il permet d'assurer précisément ce résultat. Du côté de la demande d'abord, seuls sont pris en considération les acheteurs qui sont prêts à payer le prix naturel des choses qui s'échangent, et justifient donc leur mise en vente. Du côté de l'offre, en outre, seuls sont pratiquables durablement les prix qui n'outrepassent pas leur norme naturelle, et qui s'adaptent au montant des dépenses adressées à ces choses (" effective demand»), sans attirer de concurrents supplémentaires.

A ces conditions, le prix naturel devient le véritable signifiant du prix de marché, lequel n'a de sens que dans une référence permanente à ce pivot central autour duquel il tourne à la façon des planètes autour du soleil, selon la nouvelle représentation astronomique de Newton. "Whatever may be the obstacles which hinder [the market prices. L.G.] from settling in this center of repose and continuance, they are constantly tending towards it " (W.N., I 7-75).

Le prix naturel est donc cette idée médiatrice qui permet de comprendre les différents prix observés comme appartenant au mouvement d'un même objet, dans l'espace du marché concurrentiel. La distance que met cet espace socialisant par rapport au concret des échanges est double : sont mis hors jeu d'abord tous les cas de monopoles, goulots et autres opacités qui violent la concurrence (W.N., I 7-77-79); est exclue ensuite toute modification des conditions de formation du pivot central lui-même en conséquence du mécanisme de gravitation (W.N., I 7-75).

Le marché concurrentiel fonctionne comme processus permanent d'exclusion de tout ce qui écarterait de la norme, et la multiplicité des prix observés y sont une suite d'événements accidentels en voie de normalisation. On voit que ce n'est pas tant l'existence des choses qui importe ici que la substance de ce que l'on peut en connaitre. Il n'y a pas de marché en soi sans la connaissance de sa norme (naturelle), ni bien sûr de norme sans estimation adéquate de sa valeur (réelle). C'est à ces conditions seulement que le prix de marché peut à tout instant s'imposer à tous et faire l'objet premier de la représentation du social. 


\subsection{L'espace et le temps du sujet social}

La question cruciale reste maintenant de savoir comment cette représentation de l'échange des marchandises s'applique à l'échange du travail lui-même quand celui-ci se trouve salarié. Tant que toute activité humaine n'était que travail, la médiation pouvait constituer directement une société d'égaux, où tout prix réel était automatiquement naturel, à l'instar de ce que l'on peut connaître des communautés primitives (W.N., I 8-82). Le temps et l'espace des hommes s'identifient au temps et à l'espace des choses auxquelles les hommes sont totalement soumis.

Avec la salarisation des travaux, cependant, la situation change. Car si le prix naturel s'exprime bien toujours en travail par construction de la phase initiale qui permet de représenter les échanges, tout prix réel, par contre, n'est plus forcément naturel, au sens où aucun automatisme n'impose que tous les initiateurs de l'échange soient justement récompensés. Dans une telle société, le salarié achète toute chose contre une quantité de son travail (prix réel) qui permet de rémunérer au taux normal les quantités de travail et de non-travail (prix naturel) qui ont été nécessaires pour rendre cette chose échangeable. Mais quel rapport d'échange doit-il y avoir entre le travail cédé et le travail acheté et quelle est cette juste part de rémunération qui doit revenir à chacun pour que tout le monde soit socialisé selon le même principe d'équité, y compris ceux qui ne s'impliquent pas personnellement par du travail dans les relations d'échange?

Quand on cède son travail par contrat salarial, l'équité requiert que l'on reçoive en échange un pouvoir d'acquisition sur une même quantité de travail. On ne peut sortir de cette identité formelle qu'en revenant aux origines de la division du travail (W.N., I 1-22; I 2-28), qui résulte chez Smith d'une propension à l'échange, d'un don de soi en quelque sorte plutôt que d'un calcul initial sur des besoins à satisfaire. Céder son travail, c'est donc renoncer à travailler pour soi, prendre le risque de compter sur les autres pour obtenir les nécessités et commodités nécessaires à la vie.

Le prix naturel du travail salarié devra donc être cette récompense qui donne juste le pouvoir d'acheter toutes ces choses nécessaires dont on a abandonné la production à des fins personnelles. Et le prix réel de ce travail n'est autre que la quantité de travaux qui sont actuellement cédés par ceux qui sont en train de produire ces richesses indispensables et que nous récupérons en échange de notre travail salarié.

C'est donc bien logiquement du point de vue du travailleur qui cède 
son travail qu'il faut partir pour apprécier la valeur de ce travail salarié, et non de celui qui l'achète. Le travail cédé est en soi la mesure invariante de la récompense obtenue dans l'échange contre salaire, même si « en un sens populaire » vulgarisé par les employeurs, il semble que la valeur du travail soit le prix des biens vitaux que ces derniers cèdent pour acquérir le travail en question. Car ceci est une fiction qui fait dépendre l'instrument de mesure du prix des biens vitaux eux-mêmes. Or, ce que cède le salarié " must always be the same whatever may be the quantity of goods which he receives in return for it " (W.N., I 5-50).

La question de la socialisation des non-salariés se trouve résolue du même coup, car le travail cédé mesure aussi la récompense obtenue par les employeurs. "The value which the workmen add to the materials [...] resolves itself into two parts of which one pays their wages, the other the profit of their employer upon the whole stock of materials and wages he advanced" (W.N., I 6-66). "[The master] shares in the produce of the labour of workmen, and in the share consists his profit " (W.N., I 8-83). Et Smith précise en plusieurs endroits que les rémunérations dont il parle s'entendent comme un pouvoir d'achat sur les richesses produites par les travaux salariés : "We consider [the] revenue as consisting in the power of purchasing or consuming, and not in pieces which convey it " (W.N., II 2-290; également W.N., I 6-67).

La norme du contrat salarial déterminera donc à la fois la part de richesses qui revient aux salariés et celle qui ne leur revient pas. La socialisation des non-salariés est ainsi concomitante à l'établissement du contrat salarial; c'est par la salarisation qu'ils mettent en cuvre que les employeurs se trouvent socialisés et justement rémunérés en proportion de la puissance productive du travail qu'ils ont salarié.

On voit que le prix naturel du travail est bien la médiation à travers laquelle le prix réel est repris en compte par le sujet qui se constitue socialement dans cette représentation. C'est par référence à la justice des échanges de travaux que le sujet se pense comme objet du contrat salarial, et c'est dans la prise de conscience de cet objet particulier qu'il accède au statut de sujet social. L'identification du sujet comme catégorie sociale requiert bien la médiation du prix naturel des travaux. Et on peut constater que cette procédure de socialisation permet de caractériser les dimensions spécifiques au sujet socialisé, qui interdisent de la confondre avec l'ensemble des choses qui s'échangent.

Une première dimension découle de la différenciation sociale, du fait que le fonds personnel qui assure la subsistance naturelle de chacun n'est plus le même pour tous, hors de l'état primitif. Par opposition à l'espace homogène de la marchandise, l'espace social se crée à partir d'une «distinction de rangs" entre les maîtres, d'une part, constitués des 
" classes " qui possèdent un "stock » de monnaie (marchands, industriels ou fermiers), et, d'autre part, les salariés qui n'ont que la propriété de leur travail, laquelle fonde cependant toutes les autres (W.N., I 10138).

Mais la médiation du prix naturel impose aussi une dimension temporelle qui est spécifique au sujet social, en raison même de la façon dont les mécanismes du marché viennent à la conscience quand il s'agit de travail salarié. Car le retour sur les prix nominaux est ici toujours en suspens. Cela vient de ce que la connaissance ne suffit pas pour que se constitue du sujet social, et qu'il y faut toujours aussi des actions portées par cette connaissance. Seules l'expérience et la mémoire des implications personnelles diront s'il y a ou non médiation du prix naturel dans la représentation des échanges à propos du travail salarié.

Car c'est ici l'histoire du sujet vivant qui est impliqué, et aucun mécanisme concurrentiel ne suffit plus à garantir que le concept de prix naturel se réalisera effectivement. Le retour de la norme sur les prix nominaux reste ici suspendu aux vicissitudes concrètes de l'histoire sociale, qui seules feront que le prix naturel servira ou non de médiation dans la valorisation du travail. Ceci provient de ce que le marché du travail est toujours en porte à faux par rapport aux perturbations que subissent continuellement les taux de profit et de salaire normaux, en conséquence des accroissements de la division du travail et de sa puissance productive. Il résulte de ces perturbations que le montant d'un stock donné de capital ne commande jamais une quantité naturelle (fixe) de travail (W.N., II 5-360). C'est d'ailleurs ce qui dynamise l'ensemble des échanges et ce qui confère son statut à l'économie politique en tant que science de l'enrichissement, et donc du sujet social (W.N., II 5-372; IV 0-428).

C'est aussi ce qui explique pourquoi le profit ne s'établit pas en fonction de l'état actuel de l'activité, mais de sa croissance : il est le prix à payer (en terme de travail) pour que s'opère la division du travail, sa salarisation et l'accroissement des richesses collectives. Pour autant, le profit ne s'établit pas résiduellement, comme solde de tout compte, mais il résulte d'une convention préalable sur la valeur des travaux que les employeurs vont salarier dans leur plan d'activité périodique : le contrat de salaire. Ce contrat, on le voit, ne relève pas de l'échange normal des marchandises puisqu'il anticipe sur des satisfactions virtuelles (résultant du surcroît de richesses), et qu'il confronte dans une même relation monétaire (le taux de salaire nominal) un désir de stock de capital avec un désir de fonds (de revenu).

Aucun marché du travail, aussi concurrentiel soit-il, ne peut faire que chaque échangiste se mette à la place de l'autre pour négocier la satisfac- 
tion de son désir supposé. La logique en œuvre n'est pas ici celle des confrontations instinctives, car ce n'est pas de la même chose dont chacun parle. Le sujet social est bien une construction d'une autre nature que la marchandise, qui se place directement au plan de la raison; ce qu'indique Smith à propos du salaire, quand il évoque les tarifications ou les accords sur les taux nominaux, ainsi que le caractère historique des taux naturels (W.N., V 2-870).

\subsection{L'apparente transgression de la représentation}

Au point où nous en sommes de la lecture de Smith, il apparaît bien que l'hétérogénéité des fonds de revenu et l'effet particulier de la variabilité du taux de salaire nominal dessinent un espace et une temporalité spécifiques au sujet social, qui le distinguent radicalement du monde des marchandises, dont il est pourtant issu. On vérifie, d'ailleurs, que cette identification du sujet socialisé ne peut survenir qu'à l'occasion du rapport salarial, car à l'intérieur de chacune des catégories d'employeurs ou de salariés, c'est le mécanisme de l'égalisation des taux qui seul fonctionne (W.N., I 10-116, 159). Tandis que le revenu des autres participants à la consommation nationale se greffe sur le contrat salarial, sans contribuer à la formation du prix des choses lui-même; qu'il s'agisse de l'intérêt prélevé par les prêteurs de capitaux ou des pensions dérivées des prélèvements fiscaux (W.N., I 6-70); ou qu'il s'agisse encore de la rente perçue par les propriétaires fonciers, qui font payer le droit d'utiliser leurs terres (le montant de cette rente dépend du niveau des prix, il n'en est pas la cause. W.N., I 11-162). C'est donc bien uniquement à partir du rapport salarial qu'on accède à la conscience de l'espace hétérogène de socialité (qui brise l'homothétie des relations marchandes par différenciation des rangs) et de la temporalité historique des sociétés (qui engendrent la croissance en ne répétant pas le cercle redondant des égalités formelles).

Cette genèse du sujet social reste toutefois problématique du point de vue de la théorie des représentations. Car la représentation de l'équité objective des échanges marchands ne parait jouer son rôle de médiation pour le sujet que par une prise de conscience de l'impossible respect de la norme au niveau du contrat de salaire. Et aucune procédure de rappel ne semble pouvoir théoriquement imposer la justice dans le contrat fondamental. Dès qu'un stock se trouve accumulé entre les mains de quelques-uns et que ceux-ci l'utilisent pour avancer les fonds nécessaires à la mise au travail de quelques autres, il est équitable que ces derniers reçoivent une juste « récompense », pour eux et leur famille. 
« It is but equity [...] that they who feed, cloathe and lodge the whole body of the people have such a share of the produce of their own labour as to themselves tolerably well fed, cloathed and lodged $"(W . N .$, I 8-96, également W.N., I 8-85).

Mais il est équitable aussi que celui qui a fait l'avance de son fonds dans cette opération hasardeuse et bénéfique à tous reçoive "a sufficient recompense for the trouble of employing a stock» (W.N., I 9-114).

« He could have no interest to employ [the workmen] unless he expected from the sale of their work something more than what was sufficient to replace his stock to him; and he could have no interest to employ a great stock rather a small one unless his profits were to bear some proportion to the extent of his stock 》 (W.N., I 6-66).

Mais ces profits du stock ne se règlent nullement sur la norme de travail. Leur montant correspond à ce que la division du travail a permis de produire en plus de ce qui est nécessaire à la rémunération normale des travailleurs : il est essentiellement résiduel. Or, rien n'assure, dans la seule logique des échanges marchands, que le fonds de salaire des employeurs ne franchira pas le seuil en dessous duquel il ne serait pourtant pas équitable de passer. Les employeurs, en effet, n'ont généralement pas conscience de l'intérêt qu'ils tirent eux-mêmes de la croissance; une illusion statistique les abuse régulièrement à ce sujet (la prolifération des travailleurs indépendants non enregistrés), et ils préfèrent les situations de concurrence acharnée entre les salariés. "Masters of all sorts, frequently make better bargain with their servants in dear than in cheap years [...] they naturally, therefore, command the former as more favorable for industry" (W.N., I 8-101).

Aucune société cependant ne saurait durablement se fonder sur l'injustice; bien au contraire, c'est la justice qui forme la condition minimale d'existence de la société marchande, la substance essentielle sans laquelle ne resteraient plus que des atomes individuels non socialisés et dépourvus de tout principe d'intégration (T.M.S., II 2-86). Pour que le contrat salarial assure la pérennité du sujet socialisé, il lui faut donc bien exprimer cette justice d'une façon ou d'une autre. Pour que l'image de l'échange équitable serve bien d'intermédiaire dans la socialisation des sujets, elle ne peut être logiquement transgressée. L'hypothèse d'un pouvoir politique contraignant à la justice du rapport salarial est ici hors de question, puisque l'objet social de référence, qu'on a d'abord extériorisé au départ des processus de formation des connaissances, est un principe d'équité des échanges marchands, librement mis en œuvre par la volonté de tous. Ce n'est donc pas directement le pouvoir sur le travail d'autrui qui socialise mais la justice dans les échanges de pouvoir. 
Peut-on encore éviter que l'impasse logique soit au bout du chemin des représentations? Elle se résume en trois points. Le principe de justice objectivé au départ ne semble pas pouvoir faire retour dans les sujets, motivés instinctivement par le seul appât du gain, et qui en restent là. L'abstraction représentée reste comme en suspens, incapable de susciter une quelconque procédure de réintériorisation en chacun du point de vue de l'autre. Du même coup, c'est la constitution du sujet social qui fait problème puisqu'il reste étranger à son propre principe de constitution. A défaut d'y reconnaître son image pour s'y impliquer concrètement, il demeure incapable de prendre vraiment possession de son savoir sur les choses, car l'expérience des prix de marché ne suffit pas à donner la conscience de la justice dans l'échange. Au final, c'est donc le statut de l'analyse sociale qu'on n'a pas réussi à cerner dans le mode théorique de la représentation. Si la formation des connaissances sur la vie sociale se cantonne dans une abstraction du système des marchandises, il lui manque le supplément d'âme et l'émotion nécessaire à toute connaissance véritable (en terme de représentation) : la présence sensible du sujet, désireux de se réaliser au moyen de ses propres abstractions.

\section{3. - L'ÉCONOMIE POLITIQUE COMME REPRÉSENTATION}

$\mathrm{Au}$ bout de ce chemin, trois sorties restent encore possibles. Soit la formation des connaissances en matière de société possède un statut particulier, qui la distingue de la logique des représentations propres aux autres domaines du savoir. Cette conclusion ne peut s'étayer d'aucune manière sur des références explicites à Smith; elle n'est que la conséquence logique d'une hypothèse de cohérence entre tous les textes de son œuvre. Elle clôt ici la démonstration. Soit la représentation du social imaginée par Smith n'a pas encore produit tous ses effets, pour cause d'inachèvement. Le sujet socialisé n'accédera à la conscience de son être qu'après apprentissage et mémorisation de la représentation qui est en train de s'élaborer pour la première fois à son propos. C'est la conclusion propédeutique, dont la pertinence ne peut s'apprécier que dans l'aprèsSmith. On se contentera d'effleurer cet aspect des choses car il reste encore à dire sur le texte de Smith lui-même.

Une troisième solution est, en effet, envisageable : elle consisterait à dire que cette abstraction représentée par l'échange équitable de travaux est illusoire et ne peut rendre compte de la vérité des choses. Or, cette affirmation est tout à fait testable au sein même de la logique des 
représentations dont une des règles stipule la nécessaire corrélation entre le savoir déductif et les autres formes du savoir : l'observation et le jugement. Au premier niveau, Smith émaille tout son texte d'illustrations concrètes qui semblent assez garantir le réalisme des hypothèses utilisées et la vraisemblance des résultats obtenus. Mais qu'en est-il au second niveau des savoirs qui découlent du jugement?

L'analyse de cette question va nous suggérer que la formation des connaissances sur le social, qui s'organise dans la Richesse des nations, renvoie bien à un moment émouvant où le sujet se réapproprie l'instinct de justice à travers la représentation des échanges. Ce que nous appelons l'hypothèse du moment latent. Cette hypothèse sera testée en réinterprétant la figure de "la main invisible " qui joue dans le texte de Smith le rôle d'une projection imaginaire, renvoyant à chacun l'image d'une justice à laquelle il adhère avec émotion. Cette projection ne peut provenir que d'un observateur impartial du contrat salarial, extérieur à chacun des individus qui le constitue.

\subsection{Main invisible et justice sociale}

Pour mener cette démonstration, il convient de voir d'abord comment se forme en nous l'image de la justice en général avant de s'interroger sur l'application au cas particulier de la justice sociale. L'objet fondamental de la Théorie des sentiments moraux consiste à élucider la double question de la norme des conduites, et de la façon dont elle s'impose à l'homme (T.M.S., VI 3-264). Au départ, il y a la façon dont nous jugeons les autres. Nous tenons pour "convenable " toute motivation avec laquelle nous sympathisons spontanément, au sens où notre réaction eût été la même que celle de son auteur dans des circonstances analogues. Et nous tenons pour "méritoire" toute action qui nous fait éprouver spontanément de la reconnaissance vis-à-vis de son auteur, en nous plaçant du point de vue de son destinataire.

On voit que le jugement ne relève pas essentiellement de la raison, qui est lente et incertaine (T.M.S., II 1-77); il se fonde sur l'imagination, qui autorise les transpositions individuelles, et sur le sentiment qui en émerge spontanément. Ceci nous situe exactement dans le domaine de l'instinct, au sens précédemment donné à ce mot (E.P.S., 4-168, supra). Confrontée aux données de l'observation courante, la formation du jugement est donc un mode immédiat de savoir sur les choses qui ne passe pas par la représentation. Seules, les règles générales de sa reproduction peuvent ensuite, mais ensuite seulement, être organisées par des raisonnements : ceux-ci n'en constituent pas le fondement (T.M.S., VII 3-319). 
Ayant ainsi établi le principe qui nous fait juger de la conduite des autres, il est alors possible de l'appliquer à soi-même, en se demandant si un "tranquille témoin extérieur" sympathiserait de façon impartiale avec les émotions qui nous déterminent en situation (T.M.S., III 1-110; VII 2-294). La conscience de ce mécanisme engendre le sujet vertueux, dont la motivation des actions est le désir d'être approuvé par ce témoin impartial, et qui s'en fait une norme de conduite (morale), car c'est l'objet de ses plus vifs désirs (T.M.S., II 2-83).

On notera que la formation des connaissances en matière de sujet moral obéit exactement au schéma de la représentation, avec une extériorisation, préalable de l'objet moral sous la forme du miroir (une certaine idée de l'autre nous renvoyant notre image), qui permet ensuite la constitution du sujet à partir de ce " referent secret ", par reconnaissance de l'image de soi dans le regard de l'autre. Et cette image joue bien comme médiation, car (T.M.S., VII 3-317) ce n'est pas la prime émotion qui importe dans l'établissement des normes, mais la conscience de son redoublement analogique. Ce qui est précisé dans une lettre à $\mathrm{D}$. Hume :

"In the sentiment of approbation, there are two things to be taken notice of; first the sympathetic passion of spectator; and secondly, the emotion which arises from his observing the perfect coincidence between this sympathetic passion in himself and the original passion in the person principally concerned. The last emotion, in which the sentiment of approbation properly consists, is aiways agreable and delightful " (Corresp., 43).

Dans la constitution du sujet moral, il n'y a donc pas identification à l'autre, mais identification de chacun par un retour d'images qui sont d'abord projetées sur l'autre. Et c'est dans la distance au référent que surgit l'émotion de la reconnaissance, comme par un effet de l'art. Ces principes étant établis à propos de ce qui est « convenable » au niveau de la relation de moralité interindividuelle, il est possible de montrer maintenant comment la "main invisible " transpose, au niveau social, cette main de la nature qui guide le jugement instinctif de chacun des individus moraux. Indiquons d'abord les endroits où elle apparaît dans le texte de Smith pour vérifier que les effets qu'elle provoque sont bien les expressions de la justice sociale.

Dans la Théorie des sentiments moraux, « when Providence divided the earth among a few bordmasters, it neither forgot nor abandonned those who seemed to have been left out in the partition ". La capacité des estomacs étant moindre que l'importance des désirs, les riches consomment finalement à peine plus que les pauvres... :

« they are led by an invisible hand to make nearly the same distribution of the necessaries of life, which would have been made, had the earth been 
divided into equal portions among all its inhabitants, and thus without intending it, without knowing it, advance the interest of society, and afford means of multiplication of the species " (T.M.S., IV 1-184).

Dans la Richesse des nations, chacun essaie d'engager son capital dans une activité qui lui procure le plus grand gain et la plus grande sécurité possible, sans se préoccuper de l'intérêt public qui en résulte ou non. Or, après avoir cru pouvoir montrer que le capital employé dans une activité nationale est plus productif que tout autre (W.N., II 5-371), Smith poursuit :

« upon equal or nearly equal profit, every wholesale merchant prefers that home trade to the foreign trade [...] By preferring the support of domestic to that of foreign industry, he intends only his own security; and by directing that industry in such a manner as its produce may be of the greatest value, he intends only his own gain, and he is in this, as in many cases, led by an invisible hand to promote an end which was no part of his intention " (W.N., IV 2-456).

Aux deux moments cruciaux de la dilapidation possible du fonds de salaire en consommation non productive et en exportation de capitaux, la main invisible intervient donc pour empêcher que de l'égoïsme individuel ne dérape vers de l'injustice sociale. Et une sorte d'instinct de justice supra-individuel garantit spontanément et inconsciemment que le fonds national des salaires suffira pour subvenir en chaque instant aux besoins des travailleurs concernés. Cela discrédite du même coup les révoltes égalitaires (cf. critique des violences fanatiques, T.M.S., IV 2-233, et de l' « esprit de système " des révolutionnaires français, T.M.S., IV 2-227, 234) ainsi que les protections artificielles et mercantilistes du marché national (leur critique occupe pratiquement tout le quatrième livre de la Richesse des nations). Ces opinions sont non seulement inefficaces et à la source d'un surcroît d'injustices (W.N., I 11-177), mais elles manquent, en outre, de fondement légitime.

La main invisible assure, au contraire, qu'un libre consensus social autour d'une appartenance nationale profite à tous, car c'est la croissance qui récompense cette acceptation par tous d'un juste taux de salaire national. Par les effets de justice qu'elle provoque, la main invisible figure à proprement parler la pérennité du lien social, ce sans quoi il n'existe pas. «Justice [...] is the main pillar that upholds the whole edifice. If it is removed, the great the immense fabric of human society... must in the moment crumble into atoms " (T.M.S., II 2-86). Ce faisant, la main invisible ne saurait être tenue dans le texte de Smith pour une formule de style, une habile dérobade ou même un camouflage d'intérêts particuliers. 
En tant que procédé de reconnaissance de la justice, elle est un élément indispensable à la représentation du social.

Que la main invisible soit un procédé de formation des connaissances, cela est énoncé clairement par Smith lui-même, avec la toute première évocation de cette figure qu'il propose, dans son Histoire de l'astronomie : la main invisible de Jupiter fonctionne pour le sauvage comme représentation superstitieuse des événements qu'il ne peut prévoir parce qu'ils sortent du cours naturel des choses.

" Fire burns and water refreshes, heavy bodies descend and lighter substances fly upwards, by the necessity of their own nature; nor was the invisible hand of Jupiter ever apprehended to be employed in those matters. But thunder and lightning, storms and sunshine, those more irregular events were ascribed to his favour or his anger " (E.P.S., 1-49).

Abstraction rassurante, cette main invisible de Jupiter ne suscite pourtant dans les sociétés primitives que des actions parcellaires, en raison de l'existence d'une pluralité de divinités, occupées chacune de leur propre dessein particulier. C'est avec la philosophie seulement que ces actions vont pouvoir devenir totalisantes; et circonscrire notamment un espace social par la conscience d'une complémentarité harmonieuse entre ses diverses composantes.

En résumé, la main invisible comme procédé de repérage de la justice sociale joue bien dans l'imagination le rôle du principe de cohérence, qui exorcise le chaos où retomberait toute société sans justice. Et la main invisible, comme effet spontané d'un instinct social transcendant les actions individuelles, provoque bien la sensibilité de chacun par des émotions rassurantes (déculpabilisantes, dirait-on) et stimulantes à la fois pour l'exercice concret des échanges marchands. Dans la logique même de la théorie du social en tant que représentation, la main invisible est donc un moment nécessaire à sa constitution. Encore faut-il bien préciser de quel sujet elle est la projection.

\subsection{Le sujet de la main invisible}

Jusqu'ici, l'analyse économique de Smith nous est apparue comme la représentation de la justice des échanges, objectivée d'abord dans les choses, puis servant de référence pour que des sujets sociaux se constituent dans un espace et une temporalité spécifiques. Mais le sujet de la connaissance de ce sujet social est resté hors du champ de l'analyse, et n'a pas été lui-même soumis à un quelconque processus de constitution. C'est, nous semble-t-il, ce moment de latence qu'indique la main invisible dans le mécanisme de représentation : elle montre l'existence d'un 
rapport entre l'instinct d'échange comme objet de connaissance (analysé sous l'angle des sujets sociaux) et le sujet de la connaissance de cet instinct, rapporté à son tour au sujet de ce raisonnement: un sujet inintégrable en quelque sorte, parce qu'il reste en dehors de toute objectivation de ses représentations.

Or, une telle démarche est bien repérable dans le texte de Smith, qui prend à plusieurs reprises ses distances par rapport au mécanisme social. Cela nous situe exactement dans la phase initiale de l'extériorisation (de la représentation du social) par rapport au sujet qui la pense. Un sujet qui déjoue les effets d'optique provoqués par les évidences de la croissance, dont les illusions pourtant trompent l'œil à la façon de ces représentations artistiques, qui ne mettent pas de distance suffisante avec leur modèle. Car la vanité qui fonde la course aux richesses ne procure aucun bonheur véritable (T.M.S., I 3-51, 57). "The pleasure of wealth and greatness [...] strikes the imagination as something grant and beautiful and noble [...] It is this deception which rouses and keeps in continual motion the industry of mankind" (T.M.S., V 1-183). Un sujet qui se démarque aussi de l'économie politique comme science de l'enrichissement en raison des aliénations qu'imposent au plus grand nombre ces fondements de la richesse que sont l'urbanisation et la salarialisation (W.N., I 8-101, V 1-795). Quelques indications existent aussi chez Smith permettant de suggérer par quelles médiations pourrait se constituer en retour ce sujet philosophique capable de garder ses distances par rapport à ses propres représentations : l'éducation au raisonnement et la formation à l'esthétique pourraient ainsi pallier la réduction des facultés d'abstraction et d'émotion que la croissance des richesses a provoquées (W.N., V 1-796). Cette remarque suffit déjà à indiquer que le sujet de la projection imaginaire de cette justice sociale figurée par la main invisible ne saurait être ni le sujet social, puisqu'elle reste extérieure au processus marchand, ni le sujet moral lui-même, puisqu'elle ne se réduit pas au jeu des miroirs de la relation duelle. Mais des indications plus explicites existent dans le texte de Smith à ce propos.

Que le sujet de la main invisible ne soit pas un individu social idéalisé, motivé par un instinct de justice collective, est assez clairement indiqué par tous les développements relatifs aux échanges marchands et où ne fonctionne que la recherche des intérêts particuliers. C'est, au demeurant, l'aspect doctrinal le plus connu de la Richesse des nations. Dans le passage cité sur la main invisible, par exemple, l'employeur cherche à utiliser son stock selon des perspectives de profit strictement individuel, sans que l'intérêt public entre de quelque façon en ligne de compte (également W.N., II 5-374). Quant au salarié, on ne peut en attendre davantage : " though the interest of labourer is strictly connected with 
that of society, he is incapable either of comprehending that interest, or of understanding its connection with its own " (W.N., I 11-266).

Smith met alors en garde contre les prétentions que peuvent affecter certains individus à se déterminer en fonction de l'intérêt public. "I have never known much good done by those who affected to trade for the public good" (W.N., IV 2-456). De telles prétentions conduisent d'ailleurs à des illusions trompeuses, comme l'ont bien montré les lois protectionnistes prises sous l'influence des marchands qui sont très avisés de leur intérêt particulier, et fort habiles à le présenter sous l'angle d'un intérêt général (Corresp., 272, 286). Aucun homme particulier, ni même aucun groupe d'hommes ne peut être assez sage pour juger de l'intérêt de tous les autres; et s'il en est tout de même d'assez « fous et présomptueux " pour se l'imaginer, il ne réussira qu'à perturber l' "équilibre naturel des industries ", au mépris du but recherché (W.N., IV 2-453, 630, 687).

Il reste que le sujet de la main invisible ne saurait davantage être l'individu moral, en raison de l'hétérogénéité de l'espace social. Le juste revenu de chacun ne peut s'apprécier que par rapport aux règles qui régissent son fonds particulier, et chacun doit recevoir selon les mérites de son rang, sans qu'un système de valeurs unique puisse servir de référent à tous. "In every society when the distinction of ranks has once been completely established, there have been always two different schemas or systems of morality current at the same time " (W.N., V 1794). C'est ainsi que d'un rang de la société à l'autre, une sympathie spontanée autorise le respect, voire l'admiration soit des richesses pour les uns, soit de l'innocence pour les autres (T.M.S., I 3-51, 83). Aucun jugement d'approbation ne peut s'ensuivre cependant, en raison de l'impossibilité de chacun à partager des émotions ressenties dans des conditions aussi dissemblables (T.M.S., I 5-62).

Le contrat salarial fondamental ne relève donc pas d'un échange d'émotions entre individus permutables; il n'est pas davantage un contrat marchand réglé par l'égalité des travaux échangés ; il implique la considération d'une justice distributive, en fonction d'une conscience supérieure des mérites de chacun, relativement à la place qu'il occupe dans la société. C'est avec cette distinction des mérites respectifs que chacun sympathise. Et toute offense à l'équilibre naturel des rangs (soit par avarice et ambition chez les riches, soit par paresse et luxure chez les pauvres) provoque l'indignation du spectateur impartial, ce qui fonde la punition et engendre ensuite l'institutionnalisation légale des systèmes de justice (W.N., V 1-709).

Mais l'observateur impartial des réalités interindividuelles, qui servait tout à l'heure à fonder le jugement moral, ne suffit plus. C'est ici un 
spectateur impartial et bien informé de la réalité sociale qui est requis pour porter ce jugement sur les actions et motivations de chacun des individus, qui concourent à la formation du contrat salarial. Un observateur de la réalité collective, extérieur à chacun des sujets sociaux ou moraux, et qui renvoie à tous l'image émouvante d'une justice sociale, résultant spontanément des comportements naturels de chacun, sans la médiation de quelque contrat social préalable (W.N., V 1-715). Ce spectateur impartial n'est pas autre chose qu'une représentation du sujet philosophique, transcendant pour ainsi dire ses diverses composantes, et se constituant à travers les jugements qu'il porte sur elles.

A ce stade s'ouvre la béance de la main invisible. Car si la prise de conscience de l'individu comme objet de connaissance peut bien appartenir encore au savant qui nous la représente, la conscience de cette conscience n'appartient qu'au sujet qui la désire pour lui-même, celui qui transcende toutes les images. Lorsque la formation des connaissances prend pour objet le sujet de la connaissance lui-même, la procédure des représentations reste ouverte, par construction : le moment de l'intériorisation ne peut être représenté, puisqu'il concerne l'émotion ressentie par le sujet en train d'approuver la représentation. C'est le moment impalpable du vécu, où l'affectivité et les volontés particulières du sujet déclenchent son action.

Un tel moment ne peut être conceptuellement éliminé, puisqu'il indique l'effet dynamisant que la représentation produit en retour sur le sujet. Ce moment ne peut cependant se conceptualiser et doit être seulement repéré : ici, par l'absence de mécanismes pervers dans l'échange, rendant à l'évidence injuste toute solution de type socialisant ou interventionniste. Au même titre qu'un artiste ne peut représenter l'émotion que va produire son tableau sur les spectateurs qui le regardent, de même la figure de la main invisible pointe chez Adam Smith le lieu d'apparition de cette émotion; elle mime, en quelque sorte, cette apparition qui permettra de valider l'œuvre tout entière. C'est la main de l'artiste invisible qui situe dans son œuvre même la place du lecteur acquiesçant à la présentation qui lui est faite, et la faisant sienne comme représentation.

Ce qui garantit la pérennité de la représentation du social, c'est donc un certain regard sur la représentation elle-même. Il indique ce moment émouvant où la formation de connaissances sur le moral et sur le social devient une véritable reconnaissance du sujet par lui-même. Il suit de cela que la main invisible ne saurait être une carence de la représentation, mais qu'elle doit bien rester comme une béance pour empêcher l'identification du sujet du savoir aux seules composantes sociales ou morales de son être. En ce sens, elle clôt véritablement le discours économique. En 
indiquant que la cohérence du social n'est pas représentable à l'intérieur de la sphère du social elle-même, et que la conscience de cette structure conceptuellement absente est précisément le moment où le sujet se constitue : par la conscience des artifices de sa pensée et la distance qu'il prend à l'égard de ses propres images.

\subsection{Trois enseignements pour la formation des connaissances}

Le premier enseignement de notre démonstration est que la théorie de l'échange social, telle qu'elle se présente dans la Richesse des nations, n'a pas de statut particulier par rapport aux autres formes de savoir analysées par Smith. Comme tous les « systèmes de nature ", elle est une catégorie de représentation, toujours rapportée à la conscience du sujet qui la pense, dans toutes ses dimensions.

Cette théorie part de l'observation critique des choses et n'est pas immédiatement adéquate au réel, comme l'indiquent les illusions de justice dans les prix de marché, les illusions d'accroissement du salaire quand les prix des biens vitaux augmentent, ou les illusions mêmes de la richesse matérielle par rapport au sens moral. Cette thérie construit un système d'abstractions représentant l'équité des échanges marchands qui correspond aux lois de la nature : les prix " naturels " qui sont l'image des objets tangibles représentés (les prix de marché) sont aussi des justes prix représentant l'égalité mesurable des quantités de travail qui s'échangent. Cette théorie produit son émotion propre, qui dépend de sa faculté à exciter l'imagination et de la qualité du spectacle offert. Les fondements de l'approbation sont ici la prise de conscience de la justesse de la représentation en tant que telle. Cette théorie enfin stimule l'action par la projection utopique d'un lien social spontanément réalisable à partir des seules catégories individuelles: elle constitue le sujet dans sa conscience d'être total. Au passage, on remarquera que le nouveau mode de socialisation promu par Smith, à l'encontre de la représentation qui dominait alors, en fait le premier théoricien de la dérégulation, et notamment de sa condition de possibilité comme on disait à l'époque : à savoir, l'existence d'un sujet conscient, et toujours extérieur à sa possible aliénation par quelque système social que ce soit.

Un deuxième type d'enseignement résulte de notre analyse qui concerne les rapports existant chez Adam Smith entre la Théorie des sentiments moraux et la Richesse des nations : ils passent par les Essais philosophiques. Cela nous place aux antipodes, bien sûr, des interprétations par la coupure épistémologique, à la Hildebrand (Smith est passé de l'idéalisme au matérialisme sous l'influence de son voyage en France, 
en 1766); mais cela diffère également de l'interprétation moderne (proposée par l'édition de Glasgow, T.M.S., préf., 20-25) par emboîtage conceptuel des jugements moraux et des motivations d'intérêt personnel, qui en sont une des composantes.

A la base, il y a une même disposition instinctive à l'échange qui peut concerner aussi bien les mots que les choses. Avec le travail commandé, toute marchandise vaut la quantité de travail qui permet de l'acheter (non pas celle qui est objectivée en elle); elle se définit donc d'emblée dans un rapport d'altérité, un rapport vivant à l'autre, qui est l'analogue du principe de sympathie. Quant à la gravitation des prix de marché par rapport aux prix naturels, on pourrait lui trouver une certaine analogie dans la façon dont Smith pense (T.M.S., II 2-128, 131) le rapport entre les opinions courantes de chacun et le jugement impartial d'un spectateur imaginaire. Il s'agit dans les deux cas du moment initial de l'extériorisation de l'objet de connaissance par rapport au sujet qui le pense, conformément aux principes de la représentation que l'on peut tirer des Essais philosophiques.

A peine pourrait-on suggérer que la procédure de distanciation joue plutôt sur le registre de l'imaginaire dans la Théorie des sentiments moraux et relève plutôt du type symbolique dans la Richesse des nations. Car, l'identification spéculaire à l'image de l'autre qu'on a soi-même projetée définit bien un stade narcissique, tandis que la signification du travail commandé comme désir de l'autre désigne, en effet, la marchandise, en tant que symbole. On remarquera aussi que la connaissance du sujet moral résulte des réactions du sujet à sa propre image. Ce sujet y est mis directement en scène sur le modèle des arts imitatifs et c'est en quelque sorte l'émotion qui fait ici l'objet du tableau. Quant à la connaissance sur le social, elle résulte de la mise en scène d'une "immense machinerie " que l'on contemple avec plaisir et qui excite l'imagination (T.M.S., VII 3-319), grâce à " the beauty of a systematical arrangement of different observations connected by a few common principles " (W.N., V 1-768). C'est donc à proprement parler le tableau qui fait l'objet maintenant d'une émotion.

Mais les deux représentations du social et du moral revêtent leur différence essentielle quand on approfondit le second moment logique de leur processus, celui de la réintériorisation de l'objet de connaissance, par lequel se constitue le sujet dans ses principes d'action.

Avec le sentiment d'approbation de la Théorie des sentiments moraux, toute action vaut la charge d'émotion qu'elle permet de produire. Dans la Richesse des nations, le principe de justice semble d'abord fonctionner tout seul, sans nulle part réintégrer les consciences individuelles, préoccupées seulement de leurs tractations marchandes. La main invisible se 
charge alors de rappeler la présence du sujet vivant, guidé par son instinct d'abstraction, qui lui fait comprendre que l'acte essentiel dans l'enchaînement des échanges ne peut être injuste, par construction. La sympathie se pose donc comme moment analogue et pourtant irréductible à l'échange. Irréductible, dans la mesure où le sujet social n'est pas une composante du sujet moral et que ce dernier s'autoconstitue dans sa propre représentation qui lui est suffisante. Analogue, cependant, parce que la sympathie et l'échange sont les deux composantes du sujet qui se pense dans sa totalité. Dans le monde social, la main invisible évoque donc l'exigence de sujets qui portent en eux le désir d'identification.

Une telle analyse permet, enfin, de prendre conscience du destin tragique survenu à la main invisible depuis les écrits d'Adam Smith : ce sera là notre dernier enseignement. A partir de Smith, l'économie politique tend à interpréter systématiquement la main invisible comme une métonymie (opérant une condensation de sens), qui, selon les écoles, figurerait la main du citoyen, du travailleur ou de l'État et symboliserait au choix le contrat social (main tendue), la lutte (poing fermé) ou le pouvoir (index pointé). Ces interprétations impliquent une carence originelle des textes de base et se proposent de la combler par des analyses plus approfondies. Dans sa généalogie, l'économie politique nous paraît donc s'être fourvoyée dès le départ, car la main invisible ne relève pas de la catégorie des métonymies symboliques : elle est par construction une métaphore imaginaire.

La main invisible est une métaphore (opérant transfert de sens) qui figure la justice globale en tant que référent ultime de toute la représentation; le moment latent de l'émotion provoquée par la beauté de la chose représentée, dont la démonstration est radicalement impossible et sans laquelle ne pourrait cependant se constituer aucun savoir sur les choses. Mais la métaphore est imaginaire précisément parce que cette justice du lien social ne peut être qu'évoquée, et non pas représentée dans quelque symbolisation qui impliquerait du raisonnement. L'analyse des sens externes nous a d'ailleurs appris que le toucher (de la main, donc) était à la base de la connaissance instinctive sur les choses, tandis que la vue (ici exclue) formait la base du raisonnement sur ces choses, par construction d'un rapport intelligible entre des impressions différentes (E.P.S., 4150 , supra).

Ce n'est donc pas un hasard si l'équité du lien social fondamental se trouve évoquée chez Smith par une image invisible. Celle-ci renvoie au seul moment de la révélation instinctive d'une évidence naturelle dont le sujet se reconnait partie prenante, selon sa sensibilité personnelle. Seul le sujet conscient de la justesse de la représentation peut donc en assurer la pérennité vivante dans ses passages à l'acte. Le moment magique de 
cette prise de conscience et l'émoi de son renouvellement n'ont aucune consistance permettant de le représenter : c'est le mythe fondateur du lien social, sur lequel rien d'autre ne peut être dit.

En interprétant la main invisible, non pas comme une médiation entre le sujet social et le sujet de la connaissance, mais comme un défaut d'explication qu'il s'agirait de combler abstraitement, l'économie politique modifie radicalement le statut de son savoir. Car la représentation prend elle-même la place de ce qui était jusqu'alors représenté et le lien social s'objective dans les mots qui l'ont pour la première fois rendu intelligible : c'est le discours qui devient à lui-même son propre référent, sans qu'il soit besoin d'aucun sujet extérieur pour s'en approprier le texte ${ }^{4}$. La théorie se substitue en somme à la quête vivante d'une justice utopique. L'histoire de l'analyse économique ne sera plus, dès lors, qu'un entassement de couches successives de représentations de la représentation, sans référent ultime, libéré de tout jugement extérieur au texte. Tout sujet se trouve, au contraire, directement immergé dans le réel des échanges marchands, sans qu'aucune distance ne lui assure plus la conscience de sa propre représentation. Au sens défini, précédemment à propos des arts imitatifs, il ne s'agit plus que de représentations illusoires, où le sujet social n'est que le redoublement mimétique de son modèle. On est entré dans une société spéculaire, où il n'y a plus que du sujet social, inconscient de l'être de surcroît, puisque ce statut résulte de la nature des choses que lui confère son propre discours, sans effet de médiation.

Dans l'histoire de cette représentation illusoire, c'est alors l'économie politique elle-même qui fait lien social, et sa pérennité n'est assurée que par les conditions formelles de sa reproduction, par sa propre répétition. Là où la quête du lien social faisait l'objet de la première représentation économique, c'est la conceptualisation théorique qui forme désormais le seul contenu du lien social. La forclusion du référent apparaît ainsi comme la condition initiale de la formation d'une véritable science économique sur les dépouilles du père fondateur.

En évacuant du scénario le sujet de la connaissance, conscient de la signification essentielle de ses actions sociales, les metteurs en scène de l'économie politique s'exposent toujours au risque d'un refus de reconnaissance de la part des sujets émasculés. C'est à proprement parler ce qui constitue la "crise" de l'économie politique, dont notre analyse montre que ce n'est pas un trait particulièrement spécifique de la «modernité ». La prise de conscience de ce refus a engendré, cent ans

4. Ce glissement de sens a notamment produit le soi-disant " problème de l'agrégation ». 
après, une théorie du sujet divisé (ou psychanalyse) qui rend compte de cette situation, sans pour autant donner prise sur elle. Et la théorie de la société marchande continue de multiplier depuis deux siècles ses répétitions rituelles, où il ne s'agit plus que de mimer le lien social pour donner l'illusion de son existence. Il y a beau temps, en tout cas, que la constitution du sujet n'est plus au programme.

Lucien GILLARD, C.N.R.S./Université de Paris I.

\section{ELEMENIS BIBLIOGRAPHIQUES}

Jacques DerRIDA et al., Mimesis des articulations, Paris, Flammarion, 1975.

Michel FouCAult, Les Mots et les choses. Une archéologie des sciences humaines, Paris, Gallimard, 1966.

Henri Lefebvre, La Présence et l'absence. Contributions à une théorie des représentations, Paris, Casterman, 1980.

Hannah ARENDT, Condition de l'homme moderne, Paris, Calmann-Lévy, 1961. 